\title{
Investigations on EYDFA As A Compact, Inline Amplifier
}

\author{
Nupur Agarwal \& Aditya Goel \\ Maulana Azad National Institute of Technology, Bhopal, M.P. (India)
}

\begin{abstract}
The phenomenal and continual growth in internet, the convergence of information, communication and entertainment require practical and cost-effective approaches that can be rapidly and easily deployed. Erbium Ytterbium Doped Fiber Amplifiers, an advancement over Erbium Doped Fiber Amplifiers (EDFA) are a potential candidate that can increment our high capacity/long haul links. This paper investigates some basic parameters that influence the design of EYDFA. An EYDFA in dual stage mode is configured to obtain an excellent noise figure of the order of 3-4 and uniform broadband gain flatness within $1 \mathrm{~dB}$ over a band of $30 \mathrm{~nm}$.
\end{abstract}

Keywords: - dual stage, erbium doped fiber amplifier, erbium-ytterbium doped fiber amplifier, uniform broadband gain

\section{INTRODUCTION}

Today's long-haul transmission systems represent the fourth generation utilizing multiple carrier wavelengths, which has led to an explosion of channel capacity [1,12]. Simultaneously, the changed market scenario and global success of the internet has driven the demand for higher and higher system capacity. It is accepted that "the scale and rate of bandwidth growth-driven by video, wireless data, and cloud computing are straining data centers and networks. Optical-based solutions provide the most cost-effective capacity and speed to alleviate pressures on the network." [13-14].

Presently, new dense wavelength division multiplexing (DWDM) systems that will deliver up to 1 Tbit/s of data per fiber over transoceanic distances are in the offing. This has driven the need for more efficient and possibly, more compact optical amplifiers. The Erbium Doped Fiber Amplifier is the workhorse for the current telecom. Erbium-ytterbium codoped fiber amplifier (EYDFA) which is an advancement over EDFA [1517], is also a potential contender for its efficiency and compactness since length of the active fiber is a merely 2$3 \mathrm{~m}$.

Long, repeaterless, point-to-point links represent a fast growing segment of the optical communication market, requiring practical and cost-effective approaches that can be rapidly and easily deployed. There are three possible methods to extend the reach of single-span links[1,5,7,9]: more efficient modulation and detection techniques, better forward-error correction (FEC) algorithms, and advanced optical amplification technology. While the first two methods involve redesign of the system transponder cards, the third can be implemented as an add-on to existing systems. This means the basic system can be cost-effectively designed or upgraded using standard transponders for most standard links, with advanced amplifier capability added to address only the more challenging long links.Compact and efficient optical amplifiers with large power outputs can serve as a boon here.

An optimally designed EYDFA is a promising candidate as an in-line amplifier in the $\mathrm{C}$ band. It finds application in cable television network (CATV) also. EYDFA offers significantly higher output powers than EDFAs. This results from the relatively high solubility and absorbtion coefficient of Ytterbium ions followed by an efficient energy transfer to Erbium ions via cross-relaxation in the gain medium. This work investigates the performance of EYDFA in particular the interdependence of gain, noise figure and OSNR on input signal power and frequency and also, active fiber length and presents it as a potential candidate for compact in line amplifier in wavelength division multiplexed systems. A dual stage configuration is used to obtain high gain[20] and the isolators help in achieving low noise figure.

\section{SIMULATION SETUP}

The schematic layout of the proposed dual stage EYDFA is presented in Fig. 1. The simulations were conducted. For simulations, the EYDF lengths were initially taken as $3.3 \mathrm{~m}$ and $2.0 \mathrm{~m}$ for EYDF and EYDF1 respectively. Three sets of investigations were made. In the first instance (case I), the input signal power was varied and the gain, noise figure and OSNR recorded. In the second instance (case II), the parameters were observed and analysed for varying input signal frequency. And finally in case III, simulations were made by varying length of the second stage EYDF stages and input signal power was kept fixed. An optical isolator was incorporated between the stages to eliminate any backward propagating amplified spontaneous emission (ASE). The EYDF were pumped with $1050 \mathrm{~nm}$ laser diode by using a forward pumping scheme. 


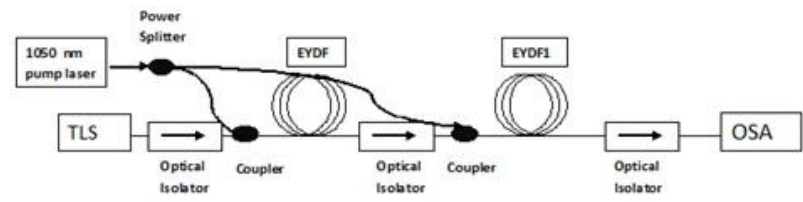

Fig. 1: Simulation setup for dual stage EYDFA

\section{Gain for varying input signal power:}

III. RESULTS AND DISCUSSIONS

The small-signal gain as a function of input signal power for a fixed EYDF and pump power is plotted in Fig. 2A for the results compiled in Table 1. The gain increases initially with an increase in input signal power and best results are obtained for input signal between $20-25 \mathrm{dBm}$. As the signal power increases a slight effect of saturation is observed. It is seen that EDFA gain decreases with the increasing signal input power. When signal power less than $-30 \mathrm{dBm}$ the amplifier works in small - signal regime where the signal gain is independent of the input signal power indeed the signal power is very weak and the amplifier works in unsaturated gain regime. When the amplifier reaches the saturation the maximum gain dropped by $3 \mathrm{~dB}$ below its unsaturated value Gmax. The physical meaning of this is the easier saturation of the EYDFA at higher signal powers for a constant pump power. The noise figure obtained is of the order of 3-4 which is good. There is little variation in gain versus input signal power for the range -10 to $-40 \mathrm{dBm}$ which implies lesser nonlinearity issues.

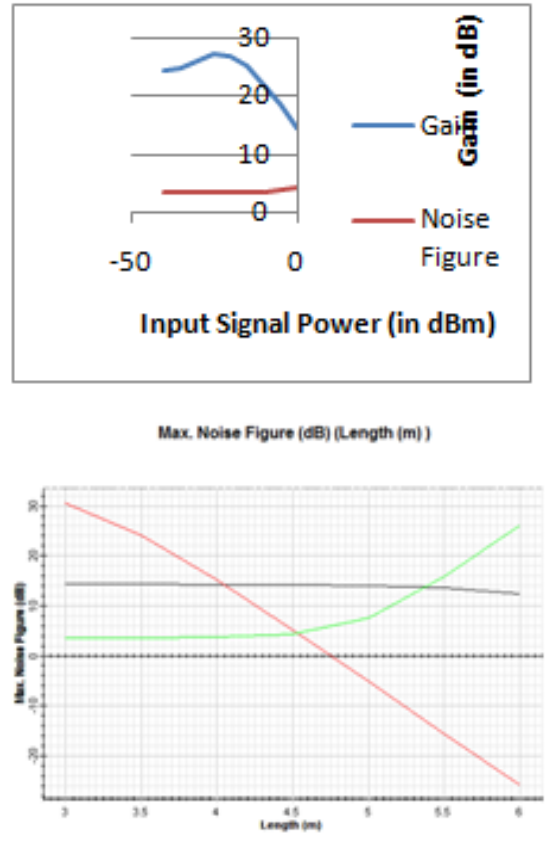

Fig: $2 \mathrm{~A}$ and $2 \mathrm{~B}$ : Gain for varying input signal power and frequency respectively

\begin{tabular}{|l|l|l|l|}
\hline $\begin{array}{l}\text { Input Signal } \\
\text { Power (in dBm) }\end{array}$ & $\begin{array}{l}\text { Gain } \\
\text { (dB) }\end{array}$ & $\begin{array}{l}\text { Noise } \\
\text { Figure }\end{array}$ & OSNR \\
\hline-40 & 24.26 & 3.4 & 14.5 \\
\hline-35 & 24.84 & 3.4 & 19.5 \\
\hline-30 & 26.04 & 3.4 & 24.5 \\
\hline-25 & 27.29 & 3.4 & 29.5 \\
\hline-20 & 27.06 & 3.4 & 34.5 \\
\hline-15 & 25.14 & 3.4 & 39.5 \\
\hline-10 & 22.13 & 3.5 & 44.4 \\
\hline-5 & 18.56 & 3.7 & 49.2 \\
\hline 0 & 14.62 & 4.3 & 53.6 \\
\hline
\end{tabular}

Table 1:Results for variation in input signal power for fixed wavelength

\section{Gain for varying EYDF length:}

The gain increases up to a certain length of fiber, and then begins to decrease after a maximum point as is evident in graph [Fig. 2B]. The decrease in gain is explained as insufficient population inversion occurs due to excessive pump depletion after a certain length and getting higher losses than the provided gain at the signal wavelength due to high total loss of Erbium doped fiber (fiber background loss+Er-Yb absorption loss). The variation of noise figure as a function of fiber length is shown. The increase in noise figure is the result of sharp pump depletion.

\section{Gain for varying input signal frequency :}

For a WDM system, a band of wavelengths is transmitted. Therefore, uniform amplification over the entire band of interest is desirable. The results are illustrated graphically in Fig. 2C. As can be seen there is very good uniformity of within $0.8 \mathrm{~dB}$ over the band even though no flattening means are being used. This results in 
no additional cost or effort for gain equalization or cheaper gain equalization if more uniformity is required. The noise figure variation is also plotted in Fig. 2D. It is clear it varies from 4 to 6 when input signal power is $0 \mathrm{dBm}$ i.e nearing saturation. NF obtained with input signal power varying between -10 to $-35 \mathrm{dBm}$ i.e. a gain excursion of $25 \mathrm{dBm}$ are uniform of the order of 3.5-4.0.
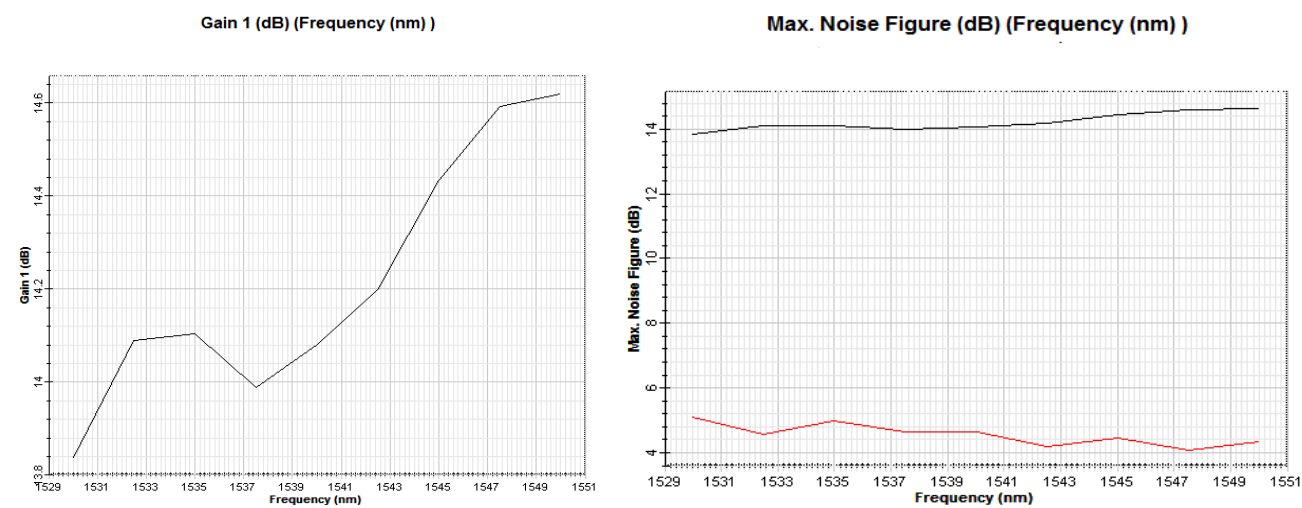

Fig. 2C and 2D : Gain and noise figure for different wavelengths of input signal for 0dB input power

\section{CONCLUSION}

An EYDFA in dual stage mode is configured to obtain an excellent noise figure of the order of 3-4 and uniform broadband gain flatness within $1 \mathrm{~dB}$ over a band of $30 \mathrm{~nm}$. The interdependence of gain, noise figure and OSNR on input signal power and frequency was analysed on basis of the simulations performed. The results obtained found in agreement with theory and other reported results. Further, the dependence of gain on length of active fiber was also investigated. Optimization of length is an important part in optimal design of EYDFA. It is clear short lengths of EYDFA in an optimally designed configuration have the potential to substitute their longer counterparts in optical communication systems. Shorter lengths of active fiber will definitely lead to more compact amplifiers and contribute to miniaturisation of devices thus improvising technology.

\section{REFERENCES}

[1] J.R. Fernandes de Oliveira, U.C.de Moura et al., "Hybrid EDFA/Raman Amplification Topology for Repeaterless $4.48 \mathrm{~Tb} / \mathrm{s}$ (40 x $112 \mathrm{~Gb} / \mathrm{s}$ DP-QPSK) Transmission Over $302 \mathrm{Km}$ of G.652 Standard Single Mode Fiber", Journal of light wave technology, vol. 31, no. 16, August 15, 2013 ,2799,

[2] Raja Ahmad, Martin Rochette, and Stephane Chatigny, "Spectrally wide and high-power Er-Yb fiber amplifier for $40 \mathrm{~Gb} / \mathrm{s}$ telecommunications Applications," OSA / CLEO/QELS 2010 of Nonlinear Optical Physics \& Materials, Vol. 21, No. 1 (2012)

[3] B. A. Hamida,, X. S. Cheng et al., "Optical amplifier with flat-gain and wideband operation utilizing highly concentrated erbium-doped fibers," Journal of Nonlinear Optical Physics \& Materials, Vol. 20, No. 4 (2011) pp.443-451

[4] M. Du et al., "Unrepeatered transmission of 107 Gb/s RZ-DQPSK over 300 km NZDSF with bi-directional Raman amplification," in Proc. OFC/NFOEC, San Diego, CA, USA, 2008

[5] D. Hardy et al., "Optics, the key to the ultra high-speed networks," in Networks: Internet, Telephony, Multimedia: Convergences and Complementarities, 1th ed. Paris, France: Springer, 2002, ch. 5, pp. 125-180.

[6] S. Bhandare et al., "Optical coherent receiver with a switchable electrical dispersion compensator for $10 \mathrm{~Gb} / \mathrm{sDPSK}$ transmission up to $300 \mathrm{~km}$ of SSMF in metro optical networks," J. Lightw. Technol., vol. 28, no. 1, pp. 47-58, Jan. 2010.

[7] D. Chang et al., "8 $120 \mathrm{~Gb} / \mathrm{s}$ unrepeatered transmission over $444 \mathrm{~km}(76.6 \mathrm{~dB})$ using distributed Raman amplification and ROPA without discrete amplification," Opt. Exp., vol. 19, no. 26, Dec. 2011.

[8] D. Mongardien et al., "2.6 Tb/s (26 $100 \mathrm{~Gb} / \mathrm{s})$ unrepeatered transmission over $401 \mathrm{~km}$ using PDM-QPSK with a coherent receiver," in Proc. ECOC, Vienna, Austria, 2009

[9] O. Bertran-Pardo et al., "Transmission of $2.6 \mathrm{~Tb} / \mathrm{s}$ using 100-Gb/s PDM-QPSK paired with a coherent receiver over a 401-km unrepeatered link," IEEE Photon. Technol. Lett., vol. 21, no. 23, pp. 1767-1769, Dec. 2009.

[10] H. Bissessur et al., "4 $100 \mathrm{~Gb} / \mathrm{s}$ unrepeatered transmission over $462 \mathrm{~km}$ using coherent PDM-QPSK format and realtime processing," in Proc. ECOC, Geneva, Switzerland, 2011

[11] Mei Du; Jianjun Yu; Xiang Zhou, "Unrepeatered Transmission of 107 Gb/s RZ-DQPSK over 300km NZDSF with Bi-directional Raman Amplification," Optical Fiber communication/National Fiber Optic Engineers Conference, vol.1, no.3, pp.24-28 Feb. 2008

[12] Raja Ahmad, Martin Rochette, and Stephane Chatigny, "Spectrally wide and high-power Er-Yb fiber amplifier for $40 \mathrm{~Gb} / \mathrm{s}$ telecommunications applications," OSA / CLEO/QELS 2010

[13] http://www.corning.com/opticalfiber/innovation/futureoffiber.aspx

[14] http://www.corning.com/news_center/news_releases/2014/2014020701.aspx 
[15] Townsend, J. E.; Barnes, W.L.; Jedrzejewski, K.P.; Grubb, S.G., "Yb3+ sensitised Er3+ doped silica optical fibre with ultrahigh transfer efficiency and gain," Electronics Letters, vol.27, no.21, pp.1958,1959, 10 Oct. 1991 doi: 10.1049/el:19911214

[16] Namkyoo Park; Wysocki, P.; Pedrazzani, R.; Grubb, S.; DiGiovanni, D.; Walker, K., "High-power Er-Yb-doped fiber amplifier with multichannel gain flatness within $0.2 \mathrm{~dB}$ over $14 \mathrm{~nm}$," Photonics Technology Letters, IEEE, vol.8, no.9, pp.1148,1150, Sept. 1996, doi: 10.1109/68.531818

[17] Park, N.; Nielsen, T.; Simpson, J.; Wysocki, P.; Pedrazzani, R.; DiGiovanni, D.; Grubb, S.; Walker, K., "High power Er-Yb linear optical fibre amplifier for CATV applications with gain tilt window over $11 \mathrm{~nm}$ and integrated dispersion pre-compensation," Electronics Letters, vol.32, no.10, pp.913,915, 9 May 1996, doi: 10.1049/el:19960592

[18] Paul F. Wysocki, Namkyoo Park and David DiGiovanni, "Dual-stage erbium-doped, erbium/ytterbium-codoped fiberamplifier with up to +26-dBmoutput power and a 17-nm flat spectrum," Vol. 21, No. 21 / November 1, 1996, Optics Letters, 1744

[19] M. Karasek, “ Optimum Design Of Erbium-Ytterbium Codoped Fibres For Large-Signal, High-Pump-Power Applications" Czechoslovak Journal of Physics, Vol. 49 (1999), No. 5

[20] S W Harun, H A Abdul Rashid, S Z Muhd-Yassin, M K Abd-Rahman, and H Ahmad, "Dual-stage Er/Yb doped fiber amplifier for gain and noise figure enhancements", IEICE Electronics Express, Vol 3, No. 23, 517-521. 